\title{
COMPUTING NON-SMOOTH MINIMIZERS WITH THE MESH TRANSFORMATION METHOD
}

\author{
JIANSONG ZHOU AND ZHIPING LI ${ }^{\dagger}$
}

\author{
LMAM \& SCHOOL OF MATHEMATICAL SCIENCES, \\ PEKING UNIVERSITY, BEIJING 100871, P.R.CHINA
}

\begin{abstract}
A regularized mesh transformation method is applied to solve a variational problem allowing a non-smooth minimizer. Since the mesh lines can be made to match the discontinuity set of the minimizer, the method efficiently improves the approximating property of the numerical solution. The error bounds dominated by the error of the energy approximation have been derived, which verify that the numerical solution obtained by the mesh transformation method is the optimal finite element solution in the sense that the corresponding error norm is minimized among all admissible mesh distributions. Numerical experiments are given to show the efficiency of the method.
\end{abstract}

\section{INTRODUCTION}

We consider numerical solutions of the problem of minimizing an elastic energy

$$
E(u ; \Omega)=\int_{\Omega} W(x, u(x), \nabla u(x)) d x
$$

in a set of admissible deformations

$$
A\left(u_{0} ; \Omega\right)=\left\{u \in W^{1, p}\left(\Omega ; \mathbb{R}^{m}\right): u=u_{0}, \text { on } \partial \Omega_{0}\right\},
$$

where $\Omega \subset R^{n}$, for $n=2$ or 3 , is a connected open set with Lipschitz continuous boundary $\partial \Omega$ and $\partial \Omega_{0} \subset \partial \Omega$ has positive $(n-1)$-dimensional measure, and $p>n$. It is well known that in general the infimum of such a problem may not be attainable when the energy density is not quasi-convex, and in such cases

1991 Mathematics Subject Classification. 65K10,65N30,73C50.

Key words and phrases. regularized mesh transformation, non-smooth minimizer, optimal finite element approximation.

The research was supported in part by NSFC major project and RFDP of China.

$\dagger$ Corresponding author: Zhiping Li, email address: lizp@math.pku.edu.cn. 
the minimizing sequences may develop finer and finer oscillations and lead to the Young measures $[1,3,4,5,10]$. The highly oscillatory functions are usually non-smooth, more precisely, they have discontinuous derivatives. Even if the energy density is convex, the problem can still have non-smooth solutions $[6$, 7]. In the computation of microstructures, one of the major difficulties for high precision numerical approximations is the presence of discontinuity, which often causes severe mesh dependence of numerical solutions $[9,11,16,18]$. To minimize the mesh dependence and to improve the numerical approximations, the mesh distribution is taken into consideration in the minimization process, which enables the mesh lines to align with the set where the derivatives are discontinuous $[14,15$, 16]. The mesh transformation method for the problem of minimizing the elastic energy $E(\cdot ; \Omega)$ in the admissible deformation set $A\left(u_{0} ; \Omega\right)$ can be introduced in the following way (for more general approach, see [16]). Let

$$
\begin{array}{r}
\mathfrak{L}(\Omega ; \Omega)=\left\{\text { bijections } L: \bar{\Omega} \rightarrow \bar{\Omega} \mid L\left(\partial \Omega_{0}\right)=\partial \Omega_{0}, L \in\left(W^{1, \infty}(\bar{\Omega})\right)^{n},\right. \\
\left.L^{-1} \in\left(W^{1, \infty}(\Omega)\right)^{n} \text {, and } \operatorname{det}(\nabla L)>0 \text {, a.e. in } \Omega\right\} .
\end{array}
$$

For $u \in W^{1, p}(\Omega ; \mathbb{R})$, define $\bar{u}(\bar{x}) \in W^{1, p}(\Omega ; \mathbb{R})$ by

$$
\bar{u}(\bar{x})=u(L(\bar{x})) ; \bar{x} \in \Omega .
$$

Then, by a change of integral variables $x \mapsto \bar{x}=L^{-1}(x)$, the energy functional $E(u, \Omega)$ is transformed to

$$
\begin{gathered}
E(u ; \Omega)=\int_{\Omega} W(x, u(x), \nabla u(x)) d x \\
=\int_{\Omega} W\left(L(\bar{x}), \bar{u}(\bar{x}), \nabla \bar{u}(\bar{x}) \cdot(\nabla L(\bar{x}))^{-1}\right) \operatorname{det}(\nabla L(\bar{x})) d \bar{x}:=E(\bar{u}, L ; \Omega) .
\end{gathered}
$$

Let $\tau_{h}$ be a family of regularized triangulations of $\Omega$ with mesh size $h$ [8], and let

$$
\begin{gathered}
T_{h}(\Omega)=\left\{L \in \mathfrak{L}(\Omega ; \Omega):\left.L\right|_{K} \text { is affine } \forall K \in \tau_{h}\right\}, \\
A_{h}\left(u_{0} ; \Omega\right)=\left\{u \in(C(\bar{\Omega}))^{m}:\left.u\right|_{K} \text { is affine } \forall K \in \tau_{h}, \text { and }\left.u\right|_{\partial \Omega}=u_{0}\right\} .
\end{gathered}
$$

Instead of minimizing the energy $E(u ; \Omega)$ in a finite element function space $A_{h}\left(u_{0} ; \Omega\right)$ defined on a fixed given mesh as in a standard finite element method, 
the mesh transformation method leads to the following discrete problem

$$
\left\{\begin{array}{l}
\text { Find }(\bar{u}, L) \in A_{h}\left(\mu_{0} ; \Omega\right) \times T_{h}(\Omega) \text { such that } \\
E(\bar{u}, L ; \Omega)=\inf _{\left(\bar{u}^{\prime}, L^{\prime}\right) \in A_{h}\left(\bar{u}_{0} ; \Omega\right) \times T_{h}(\Omega)} E\left(\bar{u}^{\prime}, L^{\prime} ; \Omega\right),
\end{array}\right.
$$

which manages to minimize the energy among all the admissible meshes defined by $L\left(\tau_{h}\right)$ for all $L \in \mathfrak{L}(\Omega ; \Omega)$. To avoid highly irregular mesh distributions from being produced in the minimization process, certain regularity techniques need to be applied [17]. The simplest way to do so is to modify the elastic energy functional $E(\bar{u}, L ; \Omega)$ by adding a mesh quality control term

$$
F_{r}(L)=\alpha \int_{\Omega}|\ln (\operatorname{det} \nabla L)|^{r} d \bar{x}
$$

where $\alpha>0$ and $r \geq 1$ are parameters, which reflect the strength of the penalty to the irregularity of the mesh distribution and need to be adjusted accordingly with the mesh refinement in computation.

In the present paper, we apply the above regularized mesh transformation method to a typical model problem, which has a non-smooth minimizer $[6,7]$. In section 2, we will introduce the model problem and discuss its solution. In section 3 , the error estimates for the finite element solutions and the corresponding stress field are derived, where the error bounds are shown to be dominated by the error of the elastic energy. Since the mesh transformation method is based upon the energy minimization principle, the result verifies that the numerical solution so obtained is the optimal finite element solution in the sense that the corresponding error norm is minimized among all admissible mesh distributions. Numerical results are presented in section 4 to show the efficiency of the method.

\section{A Model Problem and its Solution}

Consider a scalar double-well energy density

$$
W: \mathbb{R}^{n} \rightarrow \mathbb{R}, F \mapsto\left|F-F_{1}\right|^{2} \cdot\left|F-F_{2}\right|^{2},
$$

where $F_{1}=(-3,2) / \sqrt{13}$ and $F_{2}=-F_{1}$, and where $F=\nabla u$ is the deformation gradient. It is easily seen that $W \geq 0$ is non-convex, $W(F)=0$ if and only if $F=F_{1}$ or $F_{2}$. Let $W^{* *}$ be the convex envelope of $W$, we have $[6,7]$

$$
W^{* *}(F)=\left(\left(|F|^{2}-1\right)_{+}\right)^{2}+4\left(|F|^{2}-\left(F_{2} \cdot F\right)^{2}\right),
$$


where $c_{+}:=\max \{0, c\}$.

Let $\Omega=(0,1) \times\left(0, \frac{3}{2}\right)$, and let

$$
t=(3(x-1)+2 y) / \sqrt{13} \text { and } f(x, y)=-3 t^{5} / 128-t^{3} / 3 .
$$

Let $A:=u_{0}+W_{0}^{1,4}(\Omega)$ be the set of admissible deformations with $u_{0} \in W^{1,4}(\Omega)$ satisfying

$$
\left\{\begin{array}{l}
u_{0}(0, y):=\sqrt{13}(3-2 y)^{3}\left(36 y^{2}-72 y+1745\right) / 843648 \\
u_{0}(1, y):=\left(y^{3} / 39+2 y\right) / \sqrt{13} \\
u_{0}(x, 0):=9 \sqrt{13}(1-x)^{3}\left(81 x^{2}-162 x+1745\right) / 281216 \\
u_{0}\left(x, \frac{3}{2}\right):=\left(9 x^{3} / 104+3 x\right) / \sqrt{13}
\end{array}\right.
$$

Then the relaxed energy functional

$$
E^{* *}(v):=\int_{\Omega} W^{* *}(\nabla v(x)) d x+\int_{\Omega}|v(x)-f(x)|^{2} d x
$$

has a unique minimizer $u \in A$ given by $[6,7]$

$$
u(x, y)= \begin{cases}f_{0}(t+1 / 2)=-3 t^{5} / 128-t^{3} / 3, & t \leq 0, \\ f_{1}(t+1 / 2)=t^{3} / 24+t, & t \geq 0\end{cases}
$$

with the minimum energy

$$
\begin{aligned}
& E_{\text {min }}^{* *}=\int_{\Omega} W^{* *}(\nabla u(x)) d x+\int_{\Omega}|u(x)-f(x)|^{2} d x \\
& =\inf _{v \in A}\left(\int_{\Omega} W^{* *}(\nabla v(x)) d x+\int_{\Omega}|v(x)-f(x)|^{2} d x\right) \approx 0.10781476743659,
\end{aligned}
$$

and the unique minimizer $u$ is also the unique solution of the Euler-Lagrange equation

$$
\int_{\Omega} \sigma(u) \cdot \nabla v d x+2 \int_{\Omega}(u-f) v d x=0, \quad \forall v \in W_{0}^{1,4}(\Omega),
$$


where the stress field $\sigma=\sigma(u)$ is given by

$$
\sigma(u):=D W^{* *}(\nabla u)=\frac{(3,2)}{\sqrt{13}} \begin{cases}0, & t \leq 0 \\ t^{2}\left(1+3 t^{2} / 16+t^{4} / 128\right), & t \geq 0\end{cases}
$$

and the deformation gradient $\nabla u$ is given by

$$
\nabla u(x, y)=\frac{(3,2)}{\sqrt{13}} \begin{cases}-15 t^{4} / 128-t^{2}, & t \leq 0 \\ t^{2} / 8+1, & t \geq 0\end{cases}
$$

It is easily seen that $\nabla u$ is discontinuous on the line $3(x-1)+2 y=0$. However, we notice that the stress field $\sigma(u)$ is continuous. Figure 1 shows the nodal interpolation $\Pi_{h} u$ of the exact solution $u$ on a $32 \times 32$ uniform mesh, of which the mesh lines obviously cover the discontinuity set of the exact solution $u$.

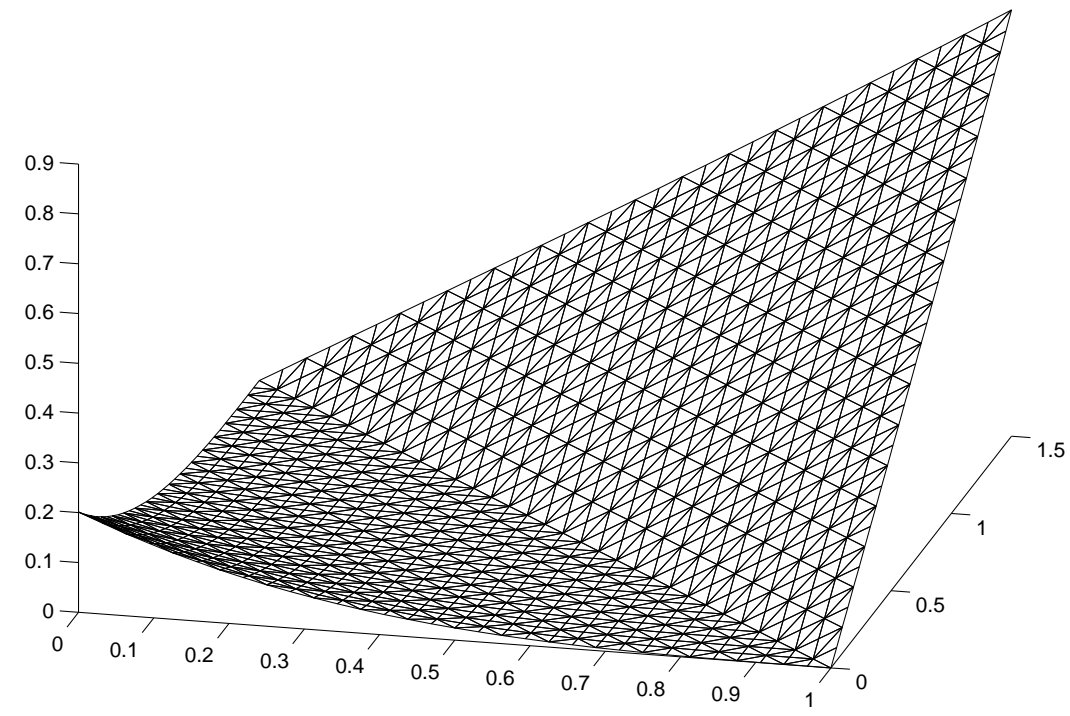

FiguRE 1. Interpolation of $u$ on a $32 \times 32$ uniform grid

\section{Discrete Problem and Its Error Estimates}

Let $\tau_{h}$ be a regular triangulation of $\Omega$ with mesh size $h[8]$. Let $\mathcal{S}^{1}\left(\tau_{h}\right)$ denote the piecewise linear conforming finite element function space

$$
\mathcal{S}^{1}\left(\tau_{h}\right):=\left\{v_{h} \in C(\bar{\Omega}):\left.v_{h}\right|_{K} \text { is affine, } \forall K \in \tau_{h}\right\} .
$$


The set of admissible deformations in the finite element space is

$$
A_{h}:=\mathcal{S}^{1}\left(\tau_{h}\right) \cap A\left(u_{0} ; \Omega\right)=u_{0 h}+\mathcal{S}_{0}^{1}\left(\tau_{h}\right),
$$

where $u_{0 h}$ is the interpolation of $u_{0}$ in $\mathcal{S}^{1}\left(\tau_{h}\right)$ and

$$
\mathcal{S}_{0}^{1}\left(\tau_{h}\right):=\left\{v_{h} \in \mathcal{S}^{1}\left(\tau_{h}\right): v_{h}=0 \text { on } \partial \Omega\right\}
$$

Then the discrete problem of the relaxation problem reads

$$
(\mathrm{DRP})\left\{\begin{array}{l}
\text { Find } u_{h} \in A_{h} \text { such that } \\
E^{* *}\left(u_{h}\right)=\inf _{v_{h} \in A_{h}} E^{* *}\left(v_{h}\right) .
\end{array}\right.
$$

Theorem 3.1. (Carstensen and Plecháč [6]) There exists a unique solution $u_{h}$ of the relaxation problem in $A_{h}$. Let $u$ denote its unique solution in $A$ and abbreviate $\sigma=D W^{* *}(\nabla u)$ and $\sigma_{h}=D W^{* *}\left(\nabla u_{h}\right)$, then the following inequality holds

$$
\left\|\sigma-\sigma_{h}\right\|_{4 / 3}^{2}+\left\|u-u_{h}\right\|_{2}^{2} \leq c \inf _{v_{h} \in A_{h}}\left(\left\|u-v_{h}\right\|_{2}^{2}+\left\|\nabla u-\nabla v_{h}\right\|_{4}^{2}\right)
$$

with a mesh-independent constant $c$.

Remark 3.1. Since the minimizer $u$ of the relaxed problem is in $W^{1,4}(\Omega)$, we have $\left\|u-\Pi_{h} u\right\|_{1,4} \rightarrow 0$ as $h \rightarrow 0$. Therefore (3.5) implies that, for the solutions of the problem (3.4), $\left\|\sigma(u)-\sigma_{h}\left(u_{h}\right)\right\|_{4 / 3}$ and $\left\|u-u_{h}\right\|_{2}$ converge to zero as $h \rightarrow 0$. Furthermore, it is not difficult to show that there exist constants $c_{1}, c_{2}>0$ such that $\max \left\{c_{1}|F|^{4}-c_{2}, 0\right\} \leq W^{* *}(F)$, and thus we have

$$
c_{1}\left\|\nabla u_{h}\right\|_{4}^{4}-c_{2} \operatorname{meas}(\Omega) \leq \int_{\Omega} W^{* *}\left(\nabla u_{h}\right) d x \leq E^{* *}\left(u_{h}\right) .
$$

On the other hand, by the Poincaré's inequality [2], $\left\|u_{h}\right\|_{1,4} \leq c_{0}\left\|\nabla u_{h}\right\|_{4}$ for all $u_{h} \in A_{h}$ with a domain dependent constant $c_{0}>0$. Hence, we have

$$
\left\|u_{h}\right\|_{1,4}^{4} \leq c_{1}^{-1} c_{0}^{4}\left(E^{* *}\left(u_{h}\right)+c_{2} \operatorname{meas}(\Omega)\right) \leq c_{1}^{-1} c_{0}^{4}\left(E^{* *}\left(\Pi_{h} u\right)+c_{2} \operatorname{meas}(\Omega)\right),
$$

where $E^{* *}\left(\Pi_{h} u\right)$ converges to $E^{* *}(u)$ as a result of the continuity of $E^{* *}(\cdot)$ and the convergence of $\Pi_{h} u$ to $u$ in $W^{1,4}(\Omega)$. This shows that $\left\|u_{h}\right\|_{1,4}$ and $\left\|\nabla u_{h}-\nabla u\right\|_{4}$ are uniformly bounded with respect to $h$, which is an important fact we will use in our error estimates. 
Theorem 3.1 provides a standard error estimate, where the error bound is measured by the interpolation error. To justify the validity of the mesh transformation method, we need to have the error bound to be measured by the error of the approximate energy. In other words, we want to show that smaller approximate energy error implies smaller error on the finite element solution and the corresponding stress. The following lemmas are useful in our analysis.

Lemma 3.1. [6] For all $F \in \mathbb{R}^{2}$, denote

$$
\xi(F):=\left(|F|^{2}-1\right)_{+} \quad \text { and } \quad \mathbb{P} F:=\left(\mathbb{I}-F_{2} F_{2}^{T}\right) F,
$$

where $\mathbb{I}$ represents the unit matrix. Define $\Sigma: \mathbb{R}^{2} \rightarrow \mathbb{R}^{2}$ by

$$
\Sigma(F):=4\left(\left(|F|^{2}-1\right)_{+} \mathbb{I}+2 \mathbb{P}\right) F, \quad \forall F \in \mathbb{R}^{2} .
$$

Then $\Sigma(F)=D W^{* *}(F)^{T}$, and for all $F, E \in \mathbb{R}^{2}$, the following inequality holds

$$
|\Sigma(F)-\Sigma(E)|^{2} \leq 8(\xi(F)+\xi(E)+2)(\Sigma(F)-\Sigma(E))^{T}(F-E) .
$$

Lemma 3.2. [6] For all $F, E \in \mathbb{R}^{2}$, we have

$$
D^{2} W(F)(E, E)=4 \xi(F)|E|^{2}+8 \mathbb{H}\left(|F|^{2}-1\right)(F \cdot E)^{2}+8\left(|E|^{2}-(F \cdot E)^{2}\right) \geq 0,
$$

Where $\mathbb{H}(\cdot)$ is the Heavyside function defined by $\mathbb{H}(x)=0$ for $x \leq 0$ and $\mathbb{H}(x)=1$ for $x>0$.

To simplify the notation, we denote $E_{h}^{* *}=\min _{v \in A_{h}} E^{* *}\left(v_{h}\right)$ and $E^{* *}=E_{m i n}^{* *}$ in the rest of this paper.

Theorem 3.2. Let $u$ and $u_{h}$ solve the relaxation problem and its discrete problem respectively, and denote $\sigma=D W^{* *}(\nabla u)$ and $\sigma_{h}=D W^{* *}\left(\nabla u_{h}\right)$. Let

$$
\begin{aligned}
& \Omega_{-}=\left\{x \in \Omega:|\nabla u| \leq 1,\left|\nabla u_{h}\right| \leq 1\right\}, \\
& \Omega_{+}=\left\{x \in \Omega:\left|\nabla u+\theta\left(\nabla u_{h}-\nabla u\right)\right| \geq 1, \forall \theta \in[0,1]\right\}, \\
& \Omega_{0}=\Omega \backslash\left\{\Omega_{-} \cup \Omega_{+}\right\} .
\end{aligned}
$$

Then, the following error estimates hold for a mesh-independent constant $c$.

$$
\begin{gathered}
E_{h}^{* *}-E^{* *} \leq \int_{\Omega}\left(\sigma_{h}-\sigma\right)\left(\nabla u_{h}-\nabla u\right) d x+\left\|u_{h}-u\right\|_{2}^{2}, \\
\left\|\sigma-\sigma_{h}\right\|_{4 / 3}^{2}+\left\|u-u_{h}\right\|_{2}^{2}+c \int_{\Omega_{0}} \int_{0}^{1}\left(\frac{11}{12}-\theta\right) G(\theta) d \theta d x \leq c\left(E_{h}^{* *}-E^{* *}\right),
\end{gathered}
$$


where $G(\theta)=D^{2} W^{* *}\left(\nabla u+\theta\left(\nabla u_{h}-\nabla u\right)\right)\left(\nabla u_{h}-\nabla u, \nabla u_{h}-\nabla u\right)$.

Proof. By the definition of $E^{* *}$ (see (2.5)), we have

$$
\begin{aligned}
& E_{h}^{* *}-E^{* *}=\int_{\Omega}\left(W^{* *}\left(\nabla u_{h}\right)-W^{* *}(\nabla u)\right) d x+\int_{\Omega}\left(\left|u_{h}-f\right|^{2}-|u-f|^{2}\right) d x \\
& =\int_{\Omega} \int_{0}^{1} D W^{* *}\left(\nabla u+t\left(\nabla u_{h}-\nabla u\right)\right)\left(\nabla u_{h}-\nabla u\right) d t d x \\
& \quad+\int_{\Omega}\left(u_{h}-u\right)\left(u_{h}+u-2 f\right) d x \\
& \quad \int_{\Omega} \int_{0}^{1}\left(D W^{* *}\left(\nabla u+t\left(\nabla u_{h}-\nabla u\right)\right)-D W^{* *}(\nabla u)\right)\left(\nabla u_{h}-\nabla u\right) d t d x \\
& \quad+\int_{\Omega} D W^{* *}(\nabla u)\left(\nabla u_{h}-\nabla u\right) d x+\int_{\Omega}\left(u_{h}-u\right)\left(u_{h}+u-2 f\right) d x \\
& \quad \int_{\Omega} \int_{0}^{1} \int_{0}^{1} t D^{2} W^{* *}\left(\nabla u+s t\left(\nabla u_{h}-\nabla u\right)\left(\nabla u_{h}-\nabla u, \nabla u_{h}-\nabla u\right) d s d t d x\right. \\
& \quad+\int_{\Omega}\left|u_{h}-u\right|^{2} d x .
\end{aligned}
$$

The last identity holds because the exact solution $u$ satisfies the Euler-Lagrange equation $[6]$

$$
\int_{\Omega} D W^{* *}(\nabla u)\left(\nabla u-\nabla u_{h}\right) d x+2 \int_{\Omega}(u-f)\left(u-u_{h}\right) d x=0 .
$$

For the first term on the right side of (3.12), we have

$$
\begin{aligned}
& \int_{\Omega} \int_{0}^{1} \int_{0}^{1} t D^{2} W^{* *}\left(\nabla u+s t\left(\nabla u_{h}-\nabla u\right)\right)\left(\nabla u_{h}-\nabla u, \nabla u_{h}-\nabla u\right) d s d t d x \\
& =\int_{\Omega} \int_{0}^{1} \int_{0}^{t} D^{2} W^{* *}\left(\nabla u+\theta\left(\nabla u_{h}-\nabla u\right)\right)\left(\nabla u_{h}-\nabla u, \nabla u_{h}-\nabla u\right) d \theta d t d x \\
& =\int_{\Omega} \int_{0}^{1} \int_{\theta}^{1} D^{2} W^{* *}\left(\nabla u+\theta\left(\nabla u_{h}-\nabla u\right)\right)\left(\nabla u_{h}-\nabla u, \nabla u_{h}-\nabla u\right) d t d \theta d x \\
& =\int_{\Omega} \int_{0}^{1}(1-\theta) D^{2} W^{* *}\left(\nabla u+\theta\left(\nabla u_{h}-\nabla u\right)\right)\left(\nabla u_{h}-\nabla u, \nabla u_{h}-\nabla u\right) d \theta d x
\end{aligned}
$$




$$
=\int_{\Omega}\left[\left(\sigma_{h}-\sigma\right)\left(\nabla u_{h}-\nabla u\right)-\int_{0}^{1} \theta G(\theta) d \theta\right] d x .
$$

Since $G(\theta) \geq 0$ by Lemma 3.2, (3.10) follows from (3.12) and (3.13).

To prove (3.11), we need to have a sharper estimate on $\int_{0}^{1} G(\theta) d \theta$. By (3.6) and (3.7), denoting $\xi_{h}=\xi\left(\nabla u_{h}\right)$ and $\xi=\xi(\nabla u)$, we have

$$
\begin{aligned}
& G(\theta)=8\left|\mathbb{P}\left(\nabla u_{h}-\nabla u\right)\right|^{2}+4\left(\left|\nabla u+\theta\left(\nabla u_{h}-\nabla u\right)\right|^{2}-1\right)_{+}\left|\nabla u_{h}-\nabla u\right|^{2} \\
& +8 \mathbb{H}\left(\left|\nabla u+\theta\left(\nabla u_{h}-\nabla u\right)\right|^{2}-1\right)\left(\left(\nabla u+\theta\left(\nabla u_{h}-\nabla u\right)\right) \cdot\left(\nabla u_{h}-\nabla u\right)\right)^{2},
\end{aligned}
$$

and

$$
\begin{aligned}
& \left(\sigma_{h}-\sigma\right)\left(\nabla u_{h}-\nabla u\right)=8\left|\mathbb{P}\left(\nabla u_{h}-\nabla u\right)\right|^{2}+4\left(\xi_{h} \nabla u_{h}-\xi \nabla u\right) \cdot\left(\nabla u_{h}-\nabla u\right) \\
& \quad=8\left|\mathbb{P}\left(\nabla u_{h}-\nabla u\right)\right|^{2}+2\left(\xi_{h}+\xi\right)\left|\nabla u_{h}-\nabla u\right|^{2}+2\left(\xi_{h}-\xi\right)\left(\left|\nabla u_{h}\right|^{2}-|\nabla u|^{2}\right) .
\end{aligned}
$$

Since, on $\Omega_{-},\left|\nabla u+\theta\left(\nabla u_{h}-\nabla u\right)\right|^{2}-1 \leq 0$ holds for all $\theta \in[0,1]$, it follows that

$$
G(\theta)=\left(\sigma_{h}-\sigma\right)\left(\nabla u_{h}-\nabla u\right)=8\left|\mathbb{P}\left(\nabla u_{h}-\nabla u\right)\right|^{2}, \quad \text { on } \Omega_{-},
$$

which leads to

$$
\int_{0}^{1} \theta G(\theta) d \theta=\frac{1}{2}\left(\sigma_{h}-\sigma\right)\left(\nabla u_{h}-\nabla u\right), \quad \text { on } \Omega_{-} .
$$

On the other hand, for all $\theta \in[0,1]$, we have

$$
\left|\nabla u+\theta\left(\nabla u_{h}-\nabla u\right)\right|^{2}-1=\theta \xi_{h}+(1-\theta) \xi+\left(\theta^{2}-\theta\right)\left|\nabla u_{h}-\nabla u\right|^{2} \geq 0, \text { on } \Omega_{+} .
$$

In particular, for $\theta=\frac{1}{3}$, this gives $\frac{1}{3}\left|\nabla u_{h}-\nabla u\right|^{2} \leq \frac{1}{2} \xi_{h}+\xi$. Thus, direct calculations lead to

$$
\begin{aligned}
& \int_{0}^{1} \theta G(\theta) d \theta=\int_{0}^{1} 8\left|\mathbb{P}\left(\nabla u_{h}-\nabla u\right)\right|^{2} \theta d \theta \\
& +\int_{0}^{1} 4 \theta\left(\theta \xi_{h}+(1-\theta) \xi+\left(\theta^{2}-\theta\right)\left|\nabla u_{h}-\nabla u\right|^{2}\right)\left|\nabla u_{h}-\nabla u\right|^{2} d \theta \\
& +\int_{0}^{1} 2 \theta\left(\left(\left|\nabla u_{h}\right|^{2}-|\nabla u|^{2}\right)^{2}+2(2 \theta-1)\left(\left|\nabla u_{h}\right|^{2}-|\nabla u|^{2}\right)\left|\nabla u_{h}-\nabla u\right|^{2}\right. \\
& \left.+(2 \theta-1)^{2}\left|\nabla u_{h}-\nabla u\right|^{4}\right) d \theta
\end{aligned}
$$




$$
\begin{aligned}
= & 4\left|\mathbb{P}\left(\nabla u_{h}-\nabla u\right)\right|^{2}+\left(\frac{4}{3} \xi_{h}+\frac{2}{3} \xi\right)\left|\nabla u_{h}-\nabla u\right|^{2}-\frac{1}{3}\left|\nabla u_{h}-\nabla u\right|^{4} \\
& +\left(\left|\nabla u_{h}\right|^{2}-|\nabla u|^{2}\right)^{2}+\frac{2}{3}\left(\left|\nabla u_{h}\right|^{2}-|\nabla u|^{2}\right)\left|\nabla u_{h}-\nabla u\right|^{2}+\frac{1}{3}\left|\nabla u_{h}-\nabla u\right|^{4} \\
\leq & 4\left|\mathbb{P}\left(\nabla u_{h}-\nabla u\right)\right|^{2}+\left(\frac{4}{3} \xi_{h}+\frac{2}{3} \xi\right)\left|\nabla u_{h}-\nabla u\right|^{2} \\
& \frac{4}{3}\left(\left|\nabla u_{h}\right|^{2}-|\nabla u|^{2}\right)^{2}+\frac{1}{3}\left|\nabla u_{h}-\nabla u\right|^{4} \\
\leq & 4\left|\mathbb{P}\left(\nabla u_{h}-\nabla u\right)\right|^{2}+\left(\frac{4}{3} \xi_{h}+\frac{2}{3} \xi\right)\left|\nabla u_{h}-\nabla u\right|^{2} \\
& +\frac{4}{3}\left(\left|\nabla u_{h}\right|^{2}-|\nabla u|^{2}\right)^{2}+\left(\frac{1}{2} \xi_{h}+\xi\right)\left|\nabla u_{h}-\nabla u\right|^{2} \\
= & 4\left|\mathbb{P}\left(\nabla u_{h}-\nabla u\right)\right|^{2}+\left(\frac{11}{6} \xi_{h}+\frac{5}{3} \xi\right)\left|\nabla u_{h}-\nabla u\right|^{2}+\frac{4}{3}\left(\left|\nabla u_{h}\right|^{2}-|\nabla u|^{2}\right)^{2} \\
\leq & \frac{11}{12}\left(\sigma_{h}-\sigma\right)\left(\nabla u_{h}-\nabla u\right), \quad \text { on } \Omega_{+} \cdot \quad(3.17)
\end{aligned}
$$

By (3.16) and (3.17), noticing that

$$
\int_{0}^{1} G(\theta) d \theta=\left(\sigma_{h}-\sigma\right)\left(\nabla u_{h}-\nabla u\right)
$$

we have

$$
\begin{aligned}
& \int_{\Omega}\left[\left(\sigma_{h}-\sigma\right)\left(\nabla u_{h}-\nabla u\right)-\int_{0}^{1} \theta G(\theta) d \theta\right] d x \\
& \quad \geq \frac{1}{12} \int_{\Omega}\left(\sigma_{h}-\sigma\right)\left(\nabla u_{h}-\nabla u\right) d x+\int_{\Omega_{0}} \int_{0}^{1}\left(\frac{11}{12}-\theta\right) G(\theta) d \theta d x .
\end{aligned}
$$

This, combined with (3.12) and (3.13), gives

$$
\begin{aligned}
\frac{1}{12} \int_{\Omega}\left(\sigma_{h}-\sigma\right)\left(\nabla u_{h}-\nabla u\right) d x & +\left\|u-u_{h}\right\|_{2}^{2} \\
& +\int_{\Omega_{0}} \int_{0}^{1}\left(\frac{11}{12}-\theta\right) G(\theta) d \theta d x \leq E_{h}^{* *}-E^{* *} .
\end{aligned}
$$


In addition, by taking $F=\nabla u_{h}$ and $E=\nabla u$ in (3.8), we obtain

$$
\left\|\sigma-\sigma_{h}\right\|_{4 / 3}^{4 / 3} \leq 4 \int_{\Omega}\left(\xi_{h}+\xi+2\right)^{2 / 3}\left(\left(\sigma_{h}-\sigma\right)\left(\nabla u_{h}-\nabla u\right)\right)^{2 / 3} d x
$$

and accordingly, by the Hölder's inequality,

$$
\left\|\sigma-\sigma_{h}\right\|_{4 / 3}^{2} \leq 8\left\|\xi_{h}+\xi+2\right\|_{2} \int_{\Omega}\left(\sigma_{h}-\sigma\right)\left(\nabla u_{h}-\nabla u\right) d x
$$

Since $\left\|\xi_{h}+\xi+2\right\|_{2}$ is uniformly bounded (see Remark 3.1), it follows from (3.19) and (3.20) that the inequality (3.11) holds for a mesh independent constant $c \geq$ $\max \left\{96\left\|\xi_{h}+\xi+2\right\|_{2}, 1\right\}$.

Remark 3.2. The error estimate (3.11) in Theorem 3.1 is not so neat because of the term $c \int_{\Omega_{0}} \int_{0}^{1}\left(\frac{11}{12}-\theta\right) G(\theta) d \theta d x$. However, for the numerical solutions in our numerical experiments, it can be verified that the set $\Omega_{0}$ is in fact empty, and thus the error estimate takes the form

$$
\left\|\sigma-\sigma_{h}\right\|_{4 / 3}^{2}+\left\|u-u_{h}\right\|_{2}^{2} \leq c\left(E_{h}^{* *}-E^{* *}\right) .
$$

Hence, in our numerical experiments, where $E_{h}^{* *}$ is minimized, (3.21) verifies that the numerical solutions obtained by the mesh transformation method are optimal in the sense that they have the least error among all admissible mesh distributions defined by $T_{h}(\Omega)$ (see $\left.(1.6)\right)$.

Theorem 3.3. Let $u$ and $u_{h}$ solve the relaxation problem and its discrete problem respectively, and denote $\sigma=D W^{* *}(\nabla u)$ and $\sigma_{h}=D W^{* *}\left(\nabla u_{h}\right)$. Then there exists a mesh independent constant $c$ such that

$$
\left\|\sigma-\sigma_{h}\right\|_{4 / 3}^{2(2+\delta)} \leq c\left(1+\frac{1}{\delta}\right)^{1+\delta}\left(E_{h}^{* *}-E^{* *}\right)\left\|\nabla u_{h}-\nabla u\right\|_{4}^{2(1+\delta)}, \quad \forall \delta \in(0,1] .
$$

Proof. By (3.6) and (3.14), denoting $\xi_{h}=\xi\left(\nabla u_{h}\right)$ and $\xi=\xi(\nabla u)$, we have by direct calculation

$$
\begin{aligned}
G(\theta) \leq & 8\left|\nabla u_{h}-\nabla u\right|^{2}+4\left(\theta \xi_{h}+(1-\theta) \xi\right)\left|\nabla u_{h}-\nabla u\right|^{2} \\
& +8\left(\left(\nabla u+\theta\left(\nabla u_{h}-\nabla u\right)\right)\left(\nabla u_{h}-\nabla u\right)\right)^{2} \\
= & 8\left|\nabla u_{h}-\nabla u\right|^{2}+4\left(\theta \xi_{h}+(1-\theta) \xi\right)\left|\nabla u_{h}-\nabla u\right|^{2}+2\left(\left|\nabla u_{h}\right|^{2}-|\nabla u|^{2}\right)^{2} \\
& +4(2 \theta-1)\left(\left|\nabla u_{h}\right|^{2}-|\nabla u|^{2}\right)\left|\nabla u_{h}-\nabla u\right|^{2}+2(2 \theta-1)^{2}\left|\nabla u_{h}-\nabla u\right|^{4}
\end{aligned}
$$




$$
\begin{aligned}
\leq & 8\left|\nabla u_{h}-\nabla u\right|^{2}+4\left(\xi_{h}+\xi\right)\left|\nabla u_{h}-\nabla u\right|^{2} \\
& +2\left(\left|\nabla u_{h}\right|^{2}-|\nabla u|^{2}\right)^{2}+\left.4|| \nabla u_{h}\right|^{2}-|\nabla u|^{2}|| \nabla u_{h}-\left.\nabla u\right|^{2}+2\left|\nabla u_{h}-\nabla u\right|^{4} \\
\leq & 8\left|\nabla u_{h}-\nabla u\right|^{2}+4\left(\xi_{h}+\xi\right)\left|\nabla u_{h}-\nabla u\right|^{2} \\
& +4\left(\left|\nabla u_{h}\right|^{2}-|\nabla u|^{2}\right)^{2}+4\left|\nabla u_{h}-\nabla u\right|^{4} \\
\leq & 8\left|\nabla u_{h}-\nabla u\right|^{2}+4\left(\xi_{h}+\xi\right)\left|\nabla u_{h}-\nabla u\right|^{2} \\
& +4\left(\left|\nabla u_{h}\right|+|\nabla u|\right)^{2}\left|\nabla u_{h}-\nabla u\right|^{2}+4\left|\nabla u_{h}-\nabla u\right|^{4} \\
\leq & 4\left(2+\xi_{h}+\xi+\left(\left|\nabla u_{h}\right|+|\nabla u|\right)^{2}+\left|\nabla u_{h}-\nabla u\right|^{2}\right)\left|\nabla u_{h}-\nabla u\right|^{2} .
\end{aligned}
$$

Define $\eta_{h}(x)=4\left(2+\xi_{h}+\xi+\left(\left|\nabla u_{h}\right|+|\nabla u|\right)^{2}+\left|\nabla u_{h}-\nabla u\right|^{2}\right)$. Let $p=2+\delta$ and $q=\frac{2+\delta}{1+\delta}$. Then, it follows that

$$
\begin{aligned}
& \left(\int_{\Omega} \int_{0}^{1} G(\theta) d \theta d x\right)^{p}=\int_{\Omega} \int_{0}^{1}((1-\theta) G(\theta))^{1 / p}(1-\theta)^{-1 / p} G(\theta)^{1 / q} d \theta d x \\
& \leq\left(\int_{\Omega} \int_{0}^{1}(1-\theta) G(\theta) d \theta d x\right)\left(\int_{\Omega} \int_{0}^{1}(1-\theta)^{-q / p} G(\theta) d \theta d x\right)^{p / q} \\
& \leq\left(E_{h}^{* *}-E^{* *}-\left\|u-u_{h}\right\|_{2}^{2}\right)\left(\int_{\Omega} \eta_{h}(x)\left|\nabla u_{h}-\nabla u\right|^{2} \int_{0}^{1}(1-\theta)^{-q / p} d \theta d x\right)^{p / q} \\
& \leq\left(E_{h}^{* *}-E^{* *}\right)\left(1+\frac{1}{\delta}\right)^{1+\delta}\left(\int_{\Omega} \eta_{h}(x)\left|\nabla u_{h}-\nabla u\right|^{2} \mathrm{~d} x\right)^{1+\delta} \\
& \leq\left\|\eta_{h}\right\|_{2}^{1+\delta}\left(1+\frac{1}{\delta}\right)^{1+\delta}\left(E_{h}^{* *}-E^{* *}\right)\left\|\nabla u_{h}-\nabla u\right\|_{4}^{2(1+\delta)}
\end{aligned}
$$

This, together with (3.18) and (3.20), gives

$$
\begin{aligned}
\left\|\sigma-\sigma_{h}\right\|_{4 / 3}^{2(2+\delta)} & \leq\left(8\left\|\xi_{h}+\xi+2\right\|_{2}\right)^{2+\delta}\left(\int_{\Omega}\left(\sigma-\sigma_{h}\right)\left(\nabla u-\nabla u_{h}\right) \mathrm{d} x\right)^{2+\delta} \\
& =\left(8\left\|\xi_{h}+\xi+2\right\|_{2}\right)^{2+\delta}\left(\int_{\Omega} \int_{0}^{1} G(\theta) d \theta d x\right)^{p} .
\end{aligned}
$$

Since $8\left\|\xi_{h}+\xi+2\right\|_{2}^{2+\delta}\left\|\eta_{h}\right\|_{2}^{1+\delta}$ is uniformly bounded for $h>0$ and $\delta \in(0,1]$ (see Remark 3.1), the conclusion of the theorem follows.

Remark 3.3. Since $\left\|\nabla u_{h}-\nabla u\right\|_{4}$ are uniformly bounded (see Remark 3.1), (3.22) literarily implies that smaller error in the energy approximation leads to smaller 
error bound in the stress field approximation, which justifies the use of the mesh transformation method.

Remark 3.4. If $u$ and $u_{h}$ are both in the space $W^{1, \infty}(\Omega)$, which is in fact confirmed by (2.10) and the numerical experiments, then the error estimate in the stress field can be rewritten as

$$
\left\|\sigma-\sigma_{h}\right\|_{4 / 3}^{2(2+\delta)} \leq c\left(1+\frac{1}{\delta}\right)^{1+\delta}\left(E_{h}^{* *}-E^{* *}\right)\left\|\nabla u_{h}-\nabla u\right\|_{2}^{2(1+\delta)},
$$

which, combined with (3.5), gives a sharper error bound than (3.22).

\section{Numerical Experiments And Results}

We start with a coarse initial mesh, for instance a $10 \times 8$ uniform mesh as shown in Figure 2, whose mesh lines do not cover the discontinuity set of the minimizer $u$, and an initial deformation $\Pi_{h} u^{0}$ (see Figure 3) with

$$
u^{0}(x, y)= \begin{cases}(1-3 x /(3+t)) u_{0}(0, y)+3 x u_{0}(x, 3 / 2) /(3+t), & t \leq 0 \\ (1-2 y /(3-t)) u_{0}(x, 0)+2 y u_{0}(1, y) /(3-t), & t \geq 0\end{cases}
$$

where $t=3 x-2 y$.

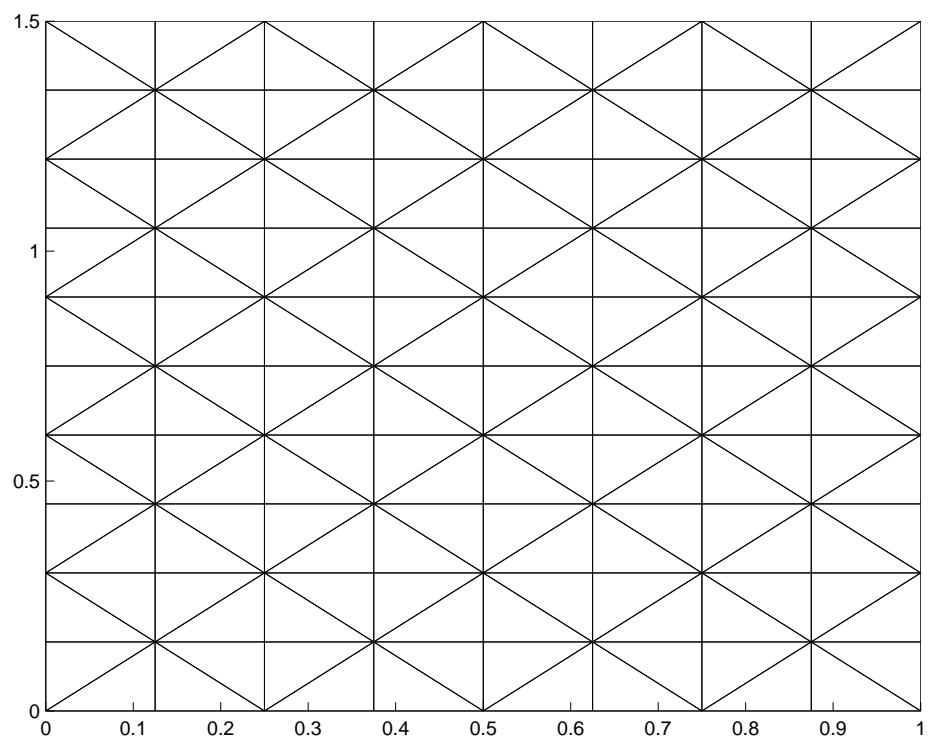

Figure 2. The $10 \times 8$ initial mesh distribution. 


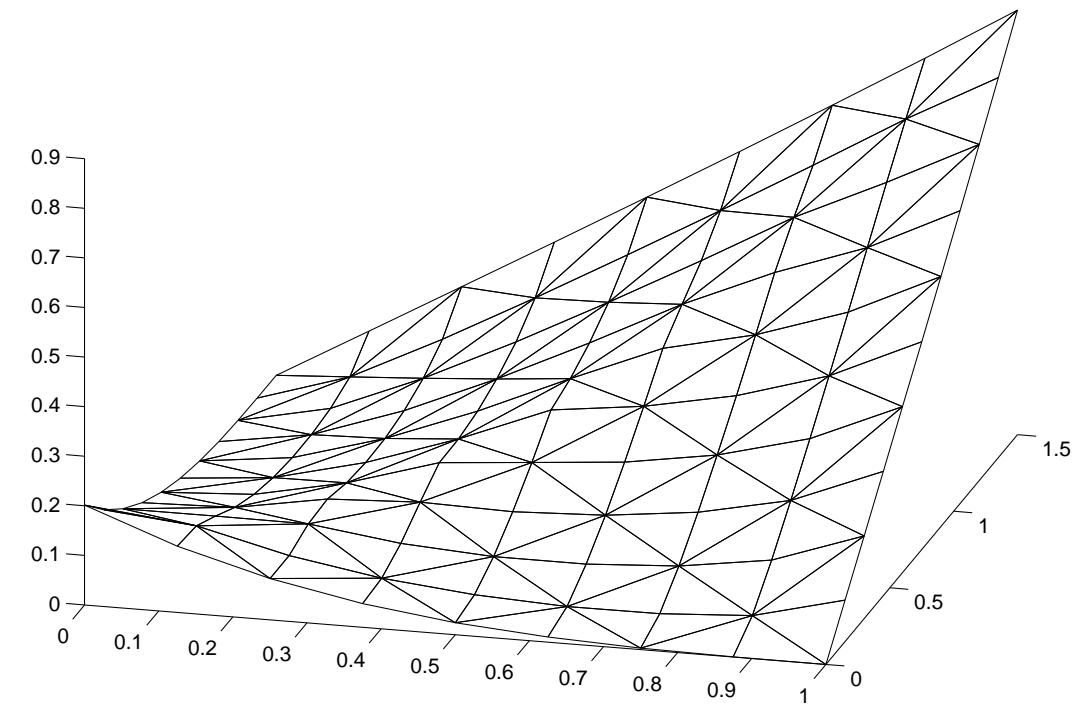

Figure 3. The initial deformation $u^{0}$.

TABLE 1. Some numerical results obtained by the regularized mesh transformation method

\begin{tabular}{|c|c|c|c|c|}
\hline$M \times N$ & $E_{h}^{* *}$ & $E_{h}^{* *}-E_{\text {min }}^{* *}$ & $F_{r}(L)$ & parameter $\alpha$ \\
\hline $10 \times 8$ & 0.1079807 & $1.6596 \times 10^{-4}$ & $2.19 \times 10^{-6}$ & $10^{-4}$ \\
\hline $20 \times 16$ & 0.1078393 & $2.4512 \times 10^{-5}$ & $3.08 \times 10^{-7}$ & $10^{-5}$ \\
\hline $40 \times 32$ & 0.1078199 & $5.1097 \times 10^{-6}$ & $4.36 \times 10^{-8}$ & $10^{-6}$ \\
\hline $80 \times 64$ & 0.1078160 & $1.2175 \times 10^{-6}$ & $5.78 \times 10^{-9}$ & $10^{-7}$ \\
\hline \hline$M \times N$ & $\left\|\sigma-\sigma_{h}\right\|_{4 / 3}$ & $\left\|u-u_{h}\right\|_{2}$ & $\left|u-u_{h}\right|_{1,4}$ & $\left|u-u_{h}\right|_{1,2}$ \\
\hline $10 \times 8$ & $3.83 \times 10^{-2}$ & $1.32 \times 10^{-3}$ & 0.4236 & 0.1813 \\
\hline $20 \times 16$ & $1.24 \times 10^{-2}$ & $3.12 \times 10^{-4}$ & 0.3640 & 0.1333 \\
\hline $40 \times 32$ & $5.19 \times 10^{-3}$ & $8.54 \times 10^{-5}$ & 0.3047 & 0.0932 \\
\hline $80 \times 64$ & $2.46 \times 10^{-3}$ & $3.75 \times 10^{-5}$ & 0.2558 & 0.0656 \\
\hline
\end{tabular}

The conjugate gradient method is applied to solve the discrete problem of the regularized mesh transformation method, where the penalty parameter $r=8$ is taken. The mesh is refined by dividing each element into four equivalent elements when certain convergence tolerance is satisfied. Some numerical results are shown 
in Table 1. The final transformed $10 \times 8$ and $20 \times 16$ meshes are shown in Figure 4 and Figure 6, and the final deformation $u_{h}$ on the corresponding meshes are shown in Figure 5 and Figure 7.

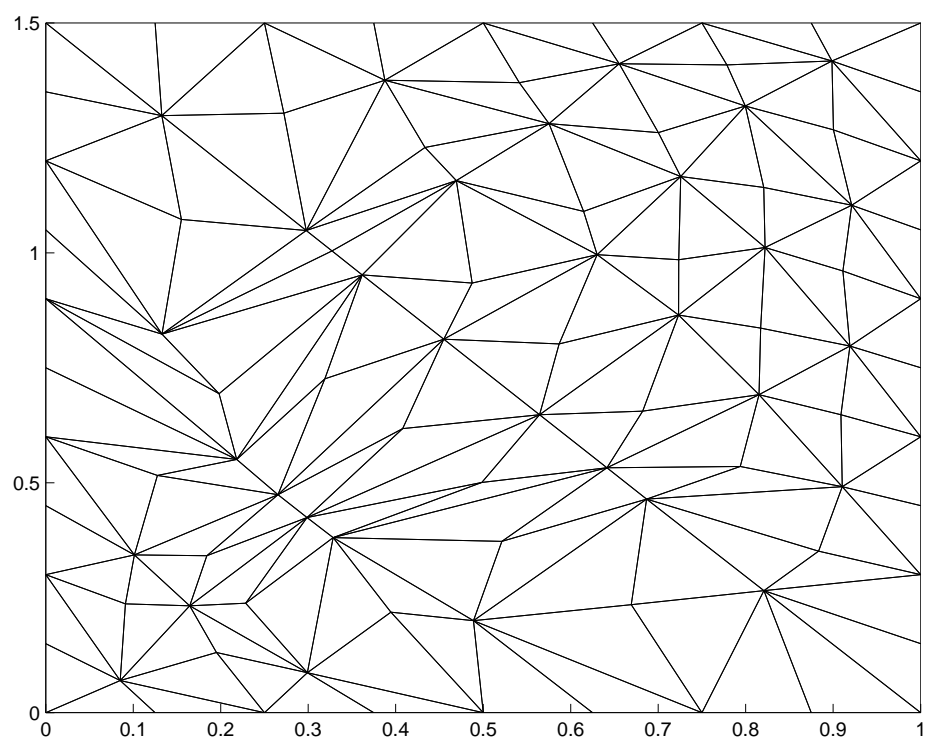

FiguRE 4 . The final $10 \times 8$ transformed mesh distribution.

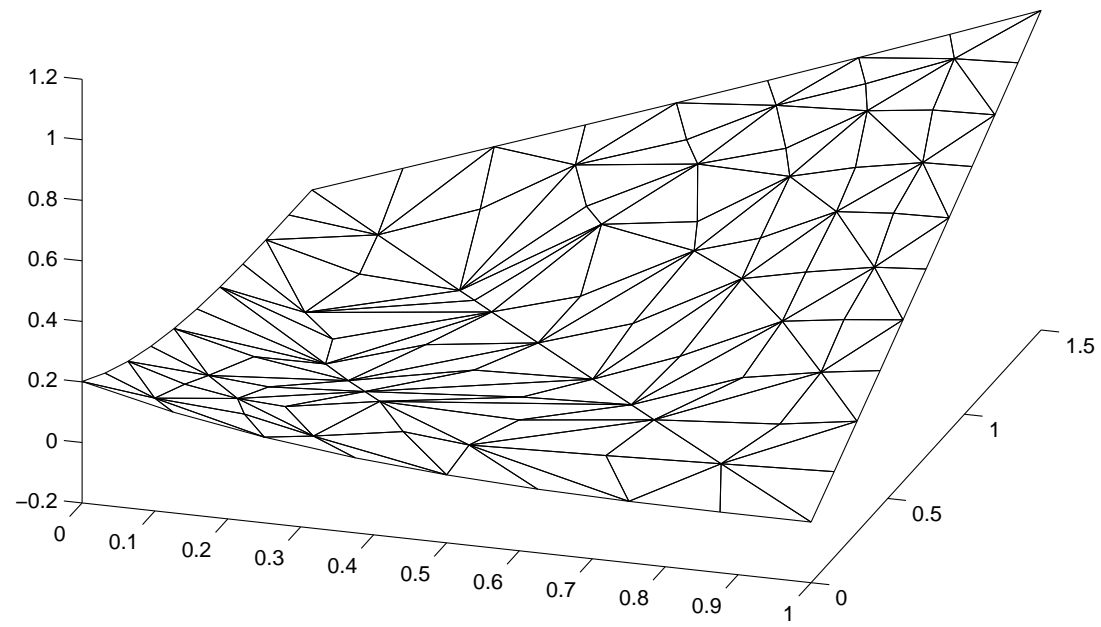

FiguRE 5. The final deformation $u_{h}$ on the $10 \times 8$ transformed mesh. 


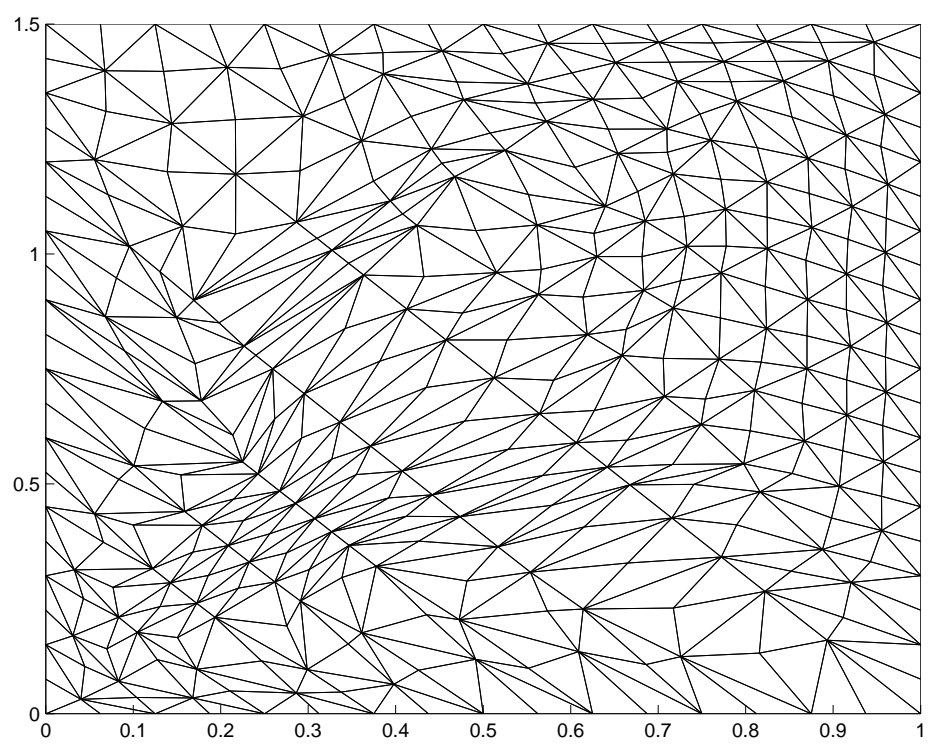

FiguRE 6 . The final $20 \times 16$ transformed mesh distribution.

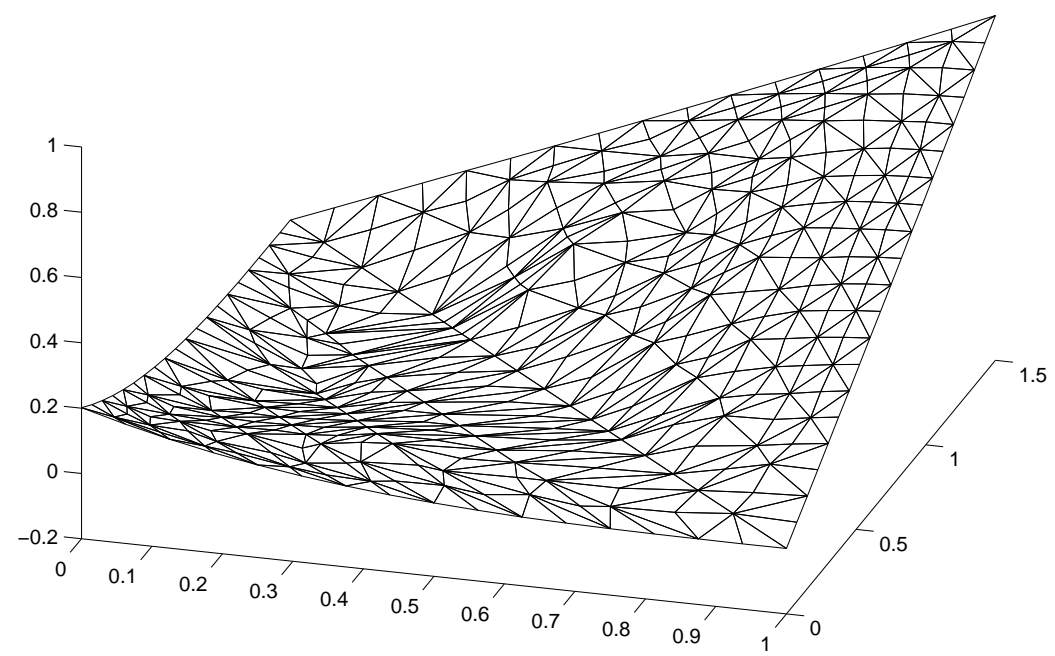

Figure 7 . The final deformation $u_{h}$ on the $20 \times 16$ transformed mesh.

It is clearly seen in Figures 4-7 that the discontinuity set $\left\{(x, y) \in \mathbb{R}^{2}\right.$ : $3(x-1)+2 y=0\}$ of the minimizer $u$ of the relaxed problem is well resolved 
by the transformed meshes' lines, which is the key contribution of the mesh transformation method to the high accuracy numerical results. In fact, as a comparison, some numerical results obtained on uniform meshes are shown in Table 2, where we can see clearly that, if it happens that the discontinuity set is covered by the mesh lines, numerical solutions with high accuracy similar to those obtained by the mesh transformation method are produced, otherwise the accuracy of the numerical solutions drop dramatically.

TABLE 2. Numerical results obtained on uniform meshes.

\begin{tabular}{|c|c|c||c|c|c|}
\hline$M \times N$ & $E_{h}^{* *}$ & $E_{h}^{* *}-E_{\text {min }}^{* *}$ & $M \times N$ & $E_{h}^{* *}$ & $E_{h}^{* *}-E_{m i n}^{* *}$ \\
\hline $10 \times 8$ & 0.1116503 & $3.84 \times 10^{-3}$ & $8 \times 8$ & 0.1086102 & $7.95 \times 10^{-4}$ \\
\hline $20 \times 16$ & 0.1089814 & $1.17 \times 10^{-3}$ & $16 \times 16$ & 0.1079301 & $1.15 \times 10^{-4}$ \\
\hline $40 \times 32$ & 0.1081967 & $3.82 \times 10^{-4}$ & $32 \times 32$ & 0.1078330 & $1.82 \times 10^{-5}$ \\
\hline $80 \times 64$ & 0.1079442 & $1.29 \times 10^{-4}$ & $64 \times 64$ & 0.1078180 & $3.22 \times 10^{-6}$ \\
\hline $160 \times 128$ & 0.1078594 & $4.46 \times 10^{-5}$ & $128 \times 128$ & 0.1078154135 & $6.46 \times 10^{-7}$ \\
\hline
\end{tabular}

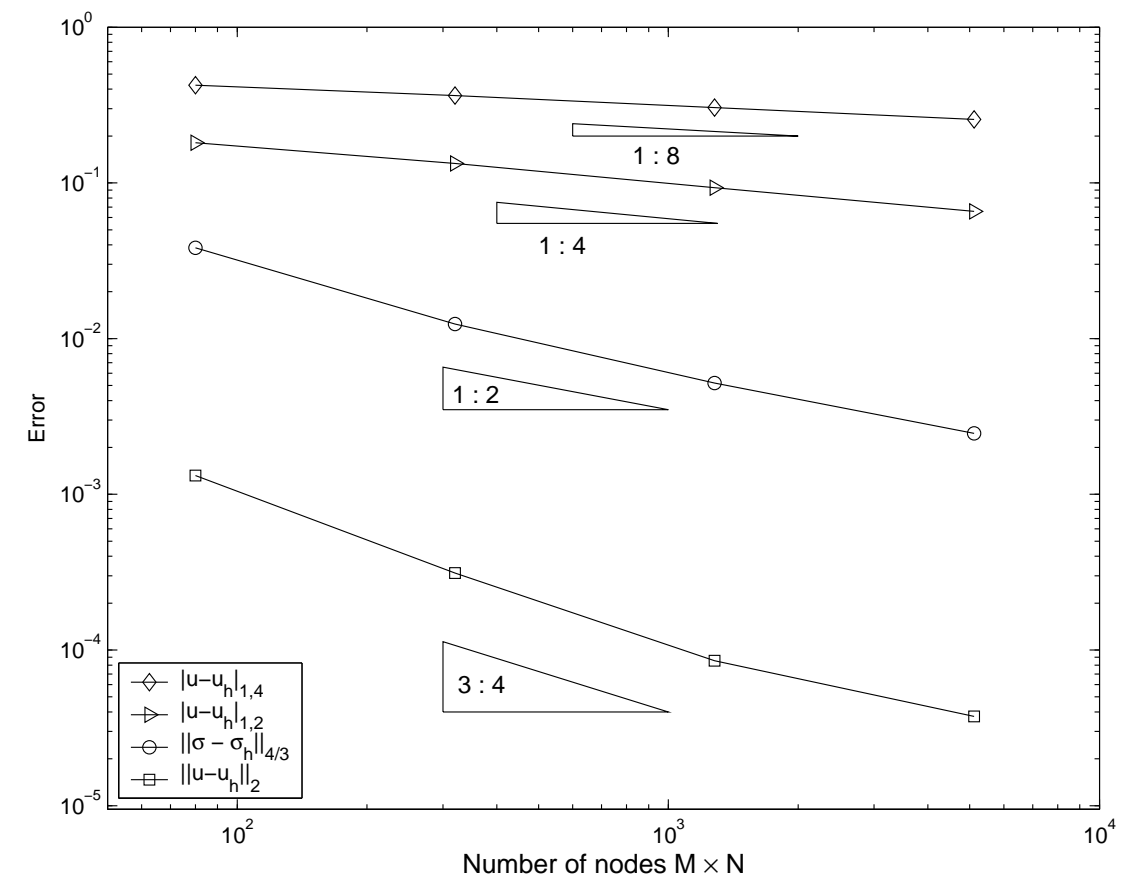

FiguRE 8 . Errors of $u_{h}$ and $\sigma_{h}$ with respect to the total node numbers. 
Figure 8 shows the relations between various errors and the total node numbers used in the computation. More precisely, let $h=\sqrt{(1.5 N)^{2}+M^{2}} /(M \cdot N)$, the numerical solutions obtained by the regularized mesh transformation method are found to have the following convergence rates:

$$
\begin{gathered}
E_{h}^{* *}-E_{\min } \propto h^{2}, \quad\left\|\sigma_{h}-\sigma\right\|_{4 / 3} \propto h, \quad\left\|u_{h}-u\right\|_{2} \propto h^{3 / 2}, \\
\left\|\nabla u_{h}-\nabla u\right\|_{4} \propto h^{1 / 4}, \quad\left\|\nabla u_{h}-\nabla u\right\|_{2} \propto h^{1 / 2}
\end{gathered}
$$

which is in nice agreement with the error estimates given in Section 3.

As we know, the mesh transformation method is a global mesh redistribution technique, while the adaptive method is a local mesh refinement technique. It is worth noticing here that the adaptive method given by Carstensen and Jochimsen [7] produces pretty much the same overall convergence rate on the stress field as the method given by the present paper. However, our numerical experiments show that the mesh transformation method is particularly efficient in the beginning of the minimization process when the discontinuous set is not yet well resolved by the mesh lines. On the other hand, the adaptive method is obviously more efficient in mesh refinement. Hence, we believe that, in general, a combination of the mesh transformation method and an adaptive method should provide a more powerful numerical method for computing discontinuous minimizers.

\section{REFERENCES}

[1] E. Acerbi and N. Fusco, Semicontinuity problems in the calculus of variations. Arch. Rat. Mech. Anal. 86(1984), 125-145.

[2] R.A. Adams, Sobolev Spaces. Academic Press, New York, 1975.

[3] J. M. Ball and R. D. James, Fine phase mixtures as minimizers of energy. Arch. Rat. Mech. Anal., 100(1987)1, 13-52.

[4] J. M. Ball and R. D. James, Proposed experimental test of a theory of fine microstructure and the two-well problem. Phil. Trans. R. Soc. London 338A(1992), 389-450.

[5] G. Buttazzo, Semicontinuity, Relaxation and Integral Representation in the Calculus of Variations. Pitman Research Notes in Mathematics Series 207. Longman, 1989.

[6] C. Carstensen and P. Plecháč, Numerical solutions of the scalar double-well problem allowing microstructure. Math. Comp., 66(1997), 997-1026. 
[7] C. Carstensen and K. Jochimsen, Adaptive finite element error control for non-convex minimization problems: Numerical two-well model example allowing microstructures. Computing, 71(2003), 175-204.

[8] P.G. Ciarlet, The Finite Element Method for Elliptic Problems. North-Holland, Amsterdam, 1978.

[9] C. Collins, M. Luskin and J. Riordan, Computational results for a two-dimensional model of crystalline microstructure, in Microstructure and Phase Transitions, IMA Volumes in Mathematics and Its Applications, Vol.54, J. Ericksen, R. James, D. Kinderlehrer and M. Luskin, eds, Springer-Verlag, New York, (1993), pp. 51-56.

[10] B. Dacorogna, Direct Methods in the Calculus of Variations, Applied Mathematical Sciences, 78, Springer-Verlag, Berlin, 1989.

[11] M.K. Gobbert and A. Prohl, A comparison of classical and new finite element methods for the computation of laminated microstructure, Appl. Numer. Math. 36(2001), pp.155178.

[12] Z. -P. Li, A theorem on lower semicontinuity of integral functionals, ESAIM: Control, Optimi. Calculus Variat, 1(1996), 169-189.

[13] Z. -P. Li, Lower semicontinuity of multiple integrals and convergent integrands, Proc. R. Soc. Edinb., 126A(1996), 363-374.

[14] Z.-P. Li, Rotational transformation method and some numerical techniques for the computation of microstructures. Math. Models Meth. Appl. Sci., 8(1998), 985-1002.

[15] Z.-P. Li, A periodic relaxation method for computing microstructures. Appl. Numer. Math., 32(2000), 291-303.

[16] Z.-P. Li, A mesh transformation method for computing microstructures. Numer. Math., 89(2001), 511-533.

[17] Z.-P. Li, Regularized mesh transformation method for computation of microstructures. Research report No.51(2003), School of Mathematical Sciences and Institute of Mathematics, Peking University.

[18] M. Luskin, On the computation of crystalline microstructure. Acta Numerica, 5(1996), 191-257.

[19] C. B. Morrey, Multiple Integrals in the Calculus of Variations, Springer, New York, 1966. 